\title{
Efecto inhibitorio en placa microbiana y propiedades antibacterianas de enjuagatorios de clorhexidina
}

\author{
ISMAEL YEVENES L \\ JUAN REYES Y \\ NESTOR CAMPOS P \\ VICTOR SARAGONI F
}

\author{
Ismael Yévenes L, Juan Reyes Y, Nestor Campos P, Víctor Saragoni \\ F. Efecto inhibitorio en placa microbiana y propiedades \\ antibacterianas de enjuagatorios de clorhexidina. Av Periodon \\ Implantol. 2003; 15, 1: 19-24.
}

\begin{abstract}
RESUMEN
El propósito de este estudio fue determinar en 20 voluntarios el efecto sobre el crecimiento de placa en 24 horas de enjuagatorios de clorhexidina, medir in vitro su actividad microbicida y determinar la concentración de clorhexidina. Tres enjuagatorios comerciales fueron usados, denominados A, B y C, los dos primeros con $0,12 \%$ y el tercero con $0,1 \%$ de clorhexidina.

El efecto sobre el crecimiento de placa en 24 horas se determino midiendo area de placa e índice de placa en tres estudios cruzados doble ciego. Las propiedades microbicidas fueron determinadas por un ensayo de inhibición del desarrollo del Streptococcus mutans, Actinomyces viscosus y Candida albicans. La determinación de clorhexidina fue realizada por cromatografía HPLC.

No se encontraron diferencias significativas en la placa formada a las 24 horas después del uso de los enjuagatorios pero menos placa se desarrollo con clorhexidina al compararla con el control. Las concentraciones de clorhexidina fueron $0,1174 \%, 0,1168 \%$ y $0,091 \%$ para $A$, B y $C$ respectivamente, valores estables después de 16 meses de elaboración. Las concentraciones en clorhexidina para A y B son valores normales mientras que el valor de C esta bajo el valor declarado. Los enjuagatorios presentan actividad microbicida contra los microorganismos usados con un tiempo de inactivación del crecimiento menor o igual a 30 segundos.

Las metodologías usadas permiten confirmar la efectividad de los enjuagatorios con diferentes concentración de clorhexidina, donde los resultados claramente sugieren que $0,1 \%$ en clorhexidina es capaz de tener actividad antiplaca y antimicrobiana cuando es usada en colutorios, no siendo necesarias concentraciones mas elevadas.
\end{abstract}

\section{PALABRAS CLAVE}

Clorhexidina; enjuagatorios; antibacteriano; antiplaca; HPLC

\section{INTRODUCCIÓN}

La clorhexidina, antiséptico bisbiguanido, es usado en clínica dental comúnmente $(1,2)$. Presenta propiedades antibacteriana y es formulado como enjuagatorio, siendo esta formulación la más empleada, presentándose en varias concentraciones $(3,4)$. EL uso de enjua- gatorios de clorhexidina es común en la población chilena.

Las revisiones bibliográficas confirman que la clorhexidina es el mas efectivo agente antiplaca $(5,6)$. La eficacia clínica esta relacionada a su naturaleza dicationica lo que permitiría explicar su actividad antimicro- 
biana prolongada por interacciones electrostáticas entre la clorhexidina y moléculas con carga en la cavidad oral $(7,8,9)$. La clorhexidina es el agente antimicrobiano bucal mas usado, efectivo y documentado en eliminar o disminuir el Estreptococos Mutans en procesos dependientes de este microorganismo $(10,11)$. La eficacia antimicrobiana de la clorhexidina permite controlar otras bacterias orales como el lactobacilo (12) siendo además efectivo en el control de hongos orales como la cándida albicans $(13,14)$

Otra de las acciones descritas para la clorhexidina es su efecto sobre la placa microbiana, donde mediciones de áreas de placa e índice de placa permiten cuantificar el efecto de la clorhexidina sobre el crecimiento de la placa en 24 horas, estas mediciones permiten con un cierto grado de certeza evaluar agentes y formulaciones antiplaca (15)

En la determinación de clorhexidina en colutorios se ha descrito el uso de la cromatografía liquida HPLC , sistema rápido, sencillo y especifico (16). Este sistema permite además determinar la clorhexidina en diferentes fluido biológicos como orina, suero, saliva con una sensibilidad bastante alta $(17,18,19,20)$.

El propósito de este estudio, fue medir el efecto sobre el crecimiento de placa microbiana en 24 horas por colutorios de clorhexidina existentes en el mercado chileno, además de determinar su concentración en clorhexidina mediante cromatografía HPLC y medir su actividad antibacteriana .

\section{MATERIALES Y MÉTODOS}

Tres marcas de enjuagatorios con clorhexidina presentes en el mercado farmacéutico chileno, Perioaid ${ }^{\circledR}$, Oralgene $^{\circledast}$, Dentilim ${ }^{\circledast}$, denominados A, B y C respectivamente, fueron usados en este estudio. Todas las muestras de enjuagatorio ( 5 de $A, 5$ de B y 6 de C), tienen diferentes fechas de fabricación y fueron obtenidos de diferentes mercados. Dos de los enjuagatorios $\mathrm{A}$ y $\mathrm{B}$ tienen la misma formulación con un 0,12\% de digluconato de clorhexidina y $\mathrm{C}$ presenta una concentración de $0,10 \%$.

Medición del efecto de la clorhexidina sobre el crecimiento de placa en 24 horas. El efecto de la clorhexidina sobre la placa microbiana se midió en tres estudios doble ciego, en 20 alumnos voluntarios y en una distribución al azar de los enjuagatorios. Los voluntarios presentaban una buena higiene oral y salud gingival, con 22 dientes medibles y sin prótesis o frenillos. En la mañana de cada estudio, los voluntarios se cepillaron con pasta de dientes y agua por dos minu- tos. Luego los dientes fueron coloreados con eritrosina y la placa residual fue removida con una copa de goma sin pasta de pulir. Los voluntarios se enjuagaron con $10 \mathrm{ml}$ por 3 minutos con el producto seleccionado con la supervisión de un investigador. La higiene oral fue suspendida por 24 horas y la placa acumulada después de ese tiempo fue revelada con eritrosina y contada por el índice de placa de Turesky y col (21) y por el índice de superficie de placa teñida de Addy y col (22) para superficies bucales de incisivos, caninos y premolares. Un periodo de espera de 7 días como mínimo se observo con una higiene normal antes de realizar los otros estudios con los otros enjuagatorios, determinando en saliva la concentración de clorhexidina residual como control. En el primer estudio se utilizo el enjuagatorio A como producto activo y como control un enjuagatorio sin clorhexidina. En el $2^{\circ}$ y $3^{\circ}$ estudio se utilizaron los enjuagatorios $\mathrm{B}$ y $\mathrm{C}$ respectivamente y el mismo colutorio control. los estudios se separaron entre si por un periodo de 4 semanas.

Métodos estadísticos. Comprobado que los índices de placa y superficie muestran una distribución normal, los datos fueron analizados para datos paramétricos utilizando el test de t de Student.

Determinación de clorhexidina. Un sistema de cromatografía líquida (HPLC) Merck Hitachi Diodo Array, con un detector fijado a $254 \mathrm{~nm}$, una columna Bondapack $\mathrm{C} 18$ de $15 \mathrm{~cm}$ como fase estacionaria y una fase móvil constituida por una mezcla de acetonitrilo agua: 50/50, $0,1 \%$ de hexasulfonato de sodio y $1 \%$ ácido acético glacial, permiten la determinación de clorhexidina.

Procedimiento. Una alicuota de 100 ul de solución estándar de clorhexidina $(0,02 \mathrm{mg} / \mathrm{ml})$ y $100 \mathrm{ul}$ de la solución problema ( $2 \mathrm{ml}$ del enjuagatorio/100ml de fase móvil), fueron inyectados en la columna. El porcentaje de clorhexidina fue calculado a través de la relación: $100(\mathrm{Au} / \mathrm{As}) /(\mathrm{Cs} / \mathrm{Cu})$, donde $\mathrm{Au}$, es el área de la clorhexidina de la solución problema, As, área de la solución estándar y Cs y Cu son las concentraciones de la clorhexidina en las soluciones estándar y problema respectivamente.

Determinación de propiedades antibacterianas. Estas fueron medidas a través de un ensayo de inhibición de desarrollo bacteriano, colocando los enjuagatorios a distintos tiempos de contacto con tres cepas de la zona bucal: Streptococcus mutans (ATCC 25175), Actinomyces viscosus (ATCC 19246) y Candida albicans (ATCC 10231). Las muestras utilizadas correspondieron a los enjuagatorios con la fecha de fabricación más antigua.

Inoculos de Prueba. S. mutans fue cultivado por 24 horas a $37^{\circ} \mathrm{C}$ en infusión de Brain Heart. A. viscosus, 


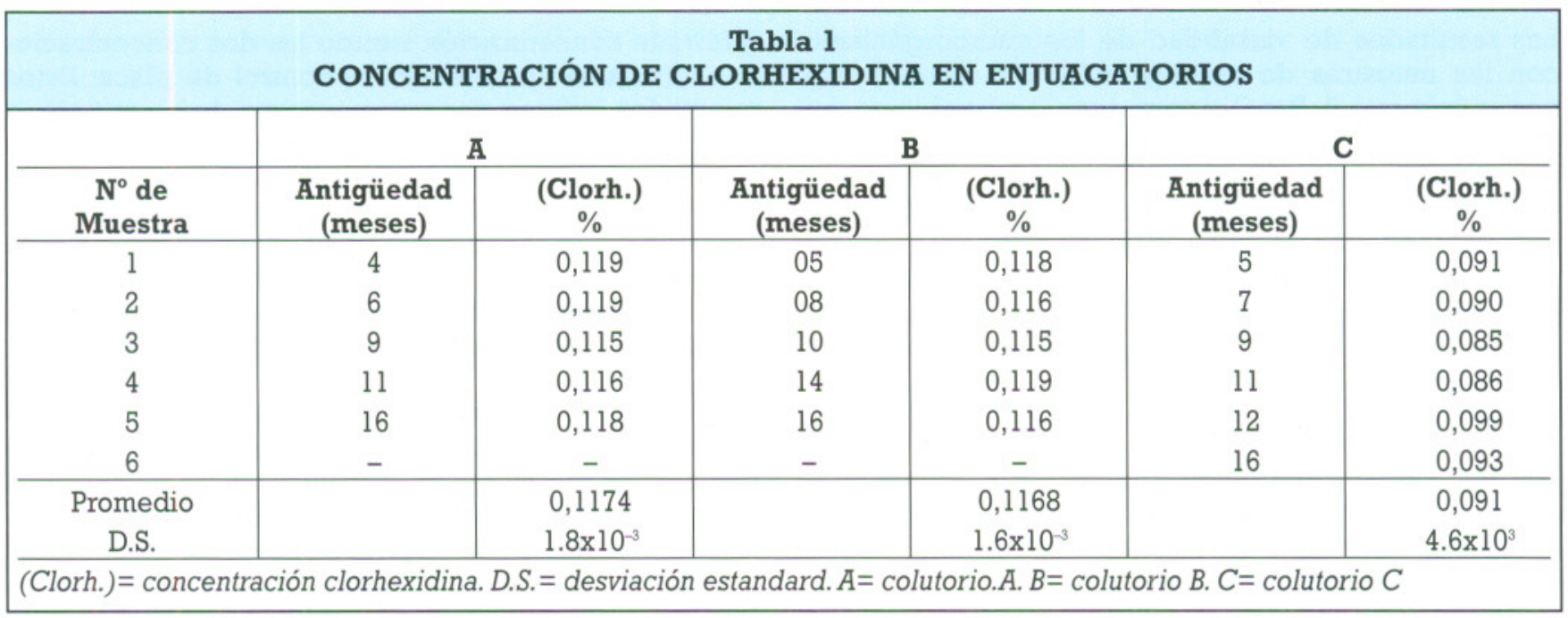

\begin{tabular}{|c|c|c|c|c|c|c|}
\hline \multicolumn{7}{|c|}{$\begin{array}{l}\text { Tabla } 2 \\
\text { PROMEDIOS Y DESVIACIONES ESTÁNDAR DE ÁREAS E ÍNDICES DE PLACA }\left(\mathrm{mm}^{2}\right) \text { SEGUIDO AL } \\
\text { USO DE COLUTORIOS CON CLORHEXIDINA }\end{array}$} \\
\hline 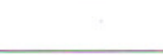 & \multicolumn{2}{|c|}{ Estudio $1(\mathbb{A})$} & \multicolumn{2}{|c|}{ Estudio 2 (B) } & \multicolumn{2}{|c|}{ Estudio 3 (C) } \\
\hline & Clorh. & Con. & Clorh. & Con. & Clorh. & Con. \\
\hline Area & 3.78 & 7.33 & 3.83 & 8.03 & 3.75 & 7.95 \\
\hline Placa & (1.28) & (2.06) & $(1.52)$ & $(2.40)$ & $(1.35)$ & (2.33) \\
\hline Indice & 1.25 & 1.95 & 1.20 & 1.81 & 1.18 & 1.88 \\
\hline Placa & $(0.23)$ & $(0.59)$ & $(0.25)$ & $(0.53)$ & $(0.35)$ & $(0.47)$ \\
\hline
\end{tabular}

cultivado por 48 horas en microaerofilia en medio de tioglicolato. C. albicans, cultivada por 24 horas a $37^{\circ} \mathrm{C}$ en caldo de Sabouraud Dextrosa.

Medio de cultivo de recuperación. Se utilizó los mismos medios del inoculo más lecitina como agente neutralizante.

Procedimiento. A $0,5 \mathrm{ml}$ del inoculo de prueba se agrego $5 \mathrm{ml}$ del enjuagatorio sin diluir, la mezcla se agito y se mantuvo a $37^{\circ} \mathrm{C}$. Se determinó la viabilidad de la mezcla inoculo-enjuagatorio a $30,60,120$ y 300 segundos después de la inoculación. Para esto se transfirió una azada de la mezcla al medio de cultivo de recuperación a los mismos tiempos. Las muestras fueron incubados a $37^{\circ} \mathrm{C}$ por 48 horas observando promoción del crecimiento microbiano.

\section{RESULTADOS}

Los resultados de los análisis de clorhexidina en los enjuagatorios son resumidos en la Tabla 1. Esta infor- ma la distribución de las muestras para las tres marcas, variando para $A$ de 4-16 meses de antigüedad, y para las muestras B y C variaron de 5-16 meses de antigüedad. Los valores promedios de clorhexidina para la marca $A$ fue de un $0,1174 \%$, para B fue de un $0,1168 \%$ y para $\mathrm{C}$ fue de un $0,091 \%$.

Medición del efecto de la clorhexidina sobre el crecimiento de placa en 24 horas. Grupos de 20 voluntarios participaron en los tres estudios, pero solo 17 alumnos participaron en los tres estudios. El promedio y la desviación estándar para los índices de placa y superficie se muestran en la Tabla 2. En los tres estudios tanto el índice de placa como el índice de superficie de placa medidos después de 24 horas tras la aplicación única del enjuagatorio son menores en los enjuagatorios con clorhexidina comparada con el control, siendo las diferencias altamente significativas $(p=0,001-0,003)$. Una comparación interestudios de los índices de placa y superficie de placa, no revela diferencias significativas entre los enjuagatorios con clorhexidina de diferentes concentraciones $(p=0,5-0,7)$, para el estudio de comparación los datos fueron analizados a través del test Kruskal-Wallis. 
Los resultados de viabilidad de los microorganismo con las muestras de enjuagatorios de clorhexidina nos señala que $A$, B y $C$, tienen acción microbicida, en las condiciones de este estudio, frente a los microorganismos testados. En todos los casos, utilizando el enjuagatorio de data mas antigua, el tiempo de inactivación fue menor o igual a 30 segundos.

\section{DISCUSIÓN}

Los resultados nos indican que la clorhexidina presente en los colutorios utilizados tienen efecto tanto sobre el crecimiento de la placa microbiana in vivo como el desarrollo bacteriano in vitro.

El uso de la cromatografía liquida HPLC permitió la determinación exacta de clorhexidina en los colutorios utilizados en la investigación clínica así como el análisis de clorhexidina residual salival en los voluntarios previo aun nuevo tratamiento. Esta metodología es de fácil aplicación, alta sensibilidad, y una reproducibilidad para un amplio rango de concentraciones de clorhexidina (23) . Las determinaciones de clorhexidina en los colutorios A y B están dentro de los limites de aceptabilidad para estos productos. Con respecto al enjuagatorio $C$, este esta bajo la norma, pero no es un problema de estabilidad de la clorhexidina, si no de la formulación que entrega una cantidad menor que la declarada.

Para visualizar los efectos de la clorhexidina en su acción microbicida, se puede ver que las cepas bacterianas seleccionadas para el estudio fueron adecuadas, porque tanto S. Mutans como A.Viscosus están presentes en la placa microbiana normal y tienen incidencia directa en la formación de la caries dental. Por otro lado, $C$. albicans esta también relacionadas a patologías comunes de la cavidad bucal. Ninguno de estos microorganismos fue capaz de crecer en presencia de los enjuagatorios que contiene clorhexidina, así organismos anaerobios (A. Viscosus) como aeróbicos (S. Mutans y C. albicans) fueron igualmente afectados por la clorhexidina confirmando su amplio espectro de acción, a pesar de la diferencia de concentración de los colutorios. Luego este tipo de enjuagatorio puede efectivamente controlar el crecimiento de bacterias y hongos orales.

Con respecto a la acción de la clorhexidina sobre el crecimiento de la placa microbiana, este estudio ha demostrado que enjuagatorios con clorhexidina son muy superiores a controles sin clorhexidina en estudios de 24 horas de duración. Pero no señala diferencias significativas en los índices de placa y superficie de placa al comparar colutorios de clorhexidina de diferente concentración siendo las dos concentraciones utilizadas efectivas en el control de placa. Estos resultados indican que estos ensayos de corto tiempo pueden ser usados en la selección de potenciales agentes o productos con acción antiplaca, usando índices convencionales de placa como el índice de area de placa de Turesky et al. (21) modificado de Quigley y Hein (24) y el índice de placa de Addy et al. (23) modificado de Shaw y Murray (25), este método tiene un alto conteo y es altamente discriminatorio.

Los resultados obtenidos con el enjuagatorio C, tanto por su efecto inhibitorio sobre el crecimiento de placa como por su efecto microbicida sobre las cepas bacterianas usadas, nos lleva a pensar que la concentración de $0,1 \%$ de digluconato de clorhexidina declarada en esta formulación, son suficientes para satisfacer las propiedades asignadas a la clorhexidina de tener actividad antibacteriana y antiplaca, e incluso obtenidas con concentraciones menores, pues la concentración promedio determinada en el colutorio C fue de $0,091 \%$. Se hace necesario entonces revisar las concentraciones de clorhexidina utilizadas en los enjuagatorios pues se podrían estar utilizando concentraciones mayores a las necesarias y por lo tanto incrementando la posibilidad de reacciones adversas a este producto.

\section{SUMMARY}

The purpose of this study was to measure plaque regrowth over $24 \mathrm{~h}$.(in vivo) and microbicidal activity (in vitro), and to determine the concentration of chlorhexidine in mouthwashes. Three commercial mouthwashes were used, $A, B$, whit $0.12 \%$ and $C$ whit 0.10 $\%$ of digluconate of chlorhexidine. The effect on $24-\mathrm{h}$ plaque regrowth were measured using of plaque area and plaque index in 3 double-blind studies in 20 subjects. For chlorhexidine concentration studies, samples all with different date of elaboration, were employed. The assessment of chlorhexidine was performed by HPLC chromatography. Microbicidal properties were measured by an inhibition assay of development of: Streptococcus mutans, Actinomyces viscosus and Candida albicans.

Not significant differences plaque reformed after using the three chlorhexidine mouthwashes but less plaque developed whit the chlorhexidine compared with control.

The chlorhexidine concentration were $0.1174 \%$, $0.1168 \%$ and $0.091 \%$ for $A, B$ and $C$ samples respectively, stability values after 16 months of elaboration respectively. Thus, A and B mouthwashes had normal 
values of chlorhexidine, while that, for $\mathrm{C}$ it would be below its declaimed value. The most dated samples of mouthrinses had microbicidal activity against the tested micro-organisms. The inactivation time of bacterial growth was lesser or equal to 30 seconds.

The methodologies applied allow to determine the effectiveness of such mouthrinses with different chlorhexidine concentration, when the results clearly suggests that $0.1 \%$ chlorhexidine is enough to get antiplaque and antimicrobial activity, when used in mouthwashes, not been necessary concentrations more high in the mouthrinses

\section{KEYWORDS}

Chlorhexidine; mouthwashes; antibacterial; plaque; HPLC

\section{CORRESPONDENCIA}

\section{Santos Dumont 964}

Santiago, Casilla 1903, Chile.

e-mail address: iyevenes@odontologia.uchile.cl

\section{REFERENCIAS}

1.Gjermo P. Chlorhexidine in dental practice. J Clin Periodontol 1974; 1: 143-52

2. Jenkins, S., Addy, M. and Newcombe, R. The effect of a chlorhexidine tooth paste on the development of plaque, gingivitis and tooth staining. J Clin Periodontol 1993; 20; $59-62$

3. Quirynen M, Avontroodt P, Peeters W, Pauwels M, Coucke W, van Steenberghe D. Effect of different chlorhexidine formulations in mouthrinses on de novo plaque formation. J Clin Periodontol 2001 Dec; 28 (12): 1127-36

4. Hoyos, D.F., Murray, J.J. and Shaw, L. The effect of chlorhexidine gel on plaque and gingivitis in Children. Br Dent J 1977; 142: 366-9

5. Lang, N.P. and Breex, M.C. Chlorhexidine digluconate, an agent for chemical plaque control and prevention of gingival inflammation. J Periodontol Res 1986; 21 (Suppl16): 74-89
6. Addy, M. and Moran, J.M. Clinical indications for the use of chemical adjuncts to plaque control: chlorhexidine formulations. Periodontol 2000 1997; 15: 52-4

7. Roberts, W.R. and Addy, M. Comparison of the in vivo an in vitro antibacterial properties of antiseptic mouthrinses containing chlorhexidine, alexidine, cetylpyridinium chloride and hexetidine; relevance to mode of action. J Clin Periodontol 1981; 8: 295-310

8. Addy, M., Jenkins, S. and Newcombe, R. The effect of some chlorhexidine containing mouthrinses on salivary bacterial counts. J Clin Periodontol 1991; 18:90-3

9. Akaho E, FukumoriY. Studies on adsorption characteristics and mechanism of adsorption of chlorhexidine mainly by carbon black.. J Pharm Sci 2001 Sep; 90 (9): 1288-97

10. Emilson, C.G. Potential efficacy of chlorhexidine against mutans streptococci and human dental caries. J Dent Res 1994; 73 (3): 682-91

11. Twetman, S., Petersson, L.G. Comparison of the efficacy of three different chlorhexidine preparations in decreasing the levels of mutans streptococci in saliva and interdental plaque. Caries Res 1998; 32 (2): 113-8

12. Cserna, A., Crist, R.L., Adams, A.B. and Dunning, D.G. Irreversible hydrocolloids: a comparison of antimicrobial efficacy. J Prosthet Dent 1994; 71 (4): 387-9

13. Schafer E, Bossmann K. Antimicrobial efficacy of chloroxylenol and chlorhexidine in the treatment of infected root canals. Am J Dent 2001 Aug; 14 (4): 233-7

14. Abu Elteen, K.H. and Whittaker, P.A. Effect of sub-inhibitory concentration of chlorhexidine on lipid and sterol composition of Candida albicans. Mycopathologia 1997; 140 (2): 69-76

15. Claydon, N., Addy, M. The use of plaque area and plaque index to measure the effect of fluoride and chlorhexidine toothpastes on 24-h plaque regrowth. J Clin Periodontol 1995; 22: 540-2

16. Bauer, M., Degude, M. and Mailhe, L. Simultaneous determination of chlorhexidine, tetracaine and their degradation products by ion-pair liquid chromatography. J Chromatogr 1984; 315: 457-64

17. Bailey, F., Brittain, P.N. and Williamson, B.F. Automated chromatographic determination of chlorhexidine in pharmaceutical preparations. J Chromatogr 1975; 109 (2): 305-12

18. Gaffney, M.H., Cooke, M. and Simpsom R. Improve methods for the determination of chlorhexidine in urine. J Chomatogr 1984; 306: 303-13 
19. Brougham, L.R., Cheng, H.Y. and Pittman, K.A. Sensitive high-performance liquid chromatographic method for the determination of chlorhexidine in human serum and urine. J Chromatogr 1986; 383 (2): 365-73

20. Lam, Y.W., Chan, D.C., Rodriguez, S.Y., Lintakoon, J.H. and lam, T.H. Sensitive high-performance liquid chromatographic assay for the determination of chlorhexidine in saliva. J Chromatogr 1993; 612 (1): 166-71

21. Turesky, S., Gilmore, N.D. and Glickman, I. Reduced plaque formation by the chlorometyl analogue of Vitamine C. J Periodontol 1970; 41: 41-3

22. Addy, M., Willis, L. and Moran, J. The effect of toothpaste and chlorhexidine rinses on plaque accumulation during a 4 day period. J Clin Periodontol 1983; 10: 89-98
23. Pesonen, T., Holmalahti, J. and Pohjola, J. Determination of chlorhexidine in saliva using high-performance liquid chromatography. J Chromatogr Biomed Appl 1995; 665 (1): $222-5$

24. Quigley, G. and Hein, J. Comparative cleansing efficency of manual and power brushing . J Am Dent Assoc 1962; 65: $26-9$

25. Shaw, L. and Murray, J. A new index for measuring extrinsic stains in clinical trials. Community Dent Oral Epidemiol 1977; 5: 116-20 